\title{
A Physically Based Theoretical Model of Spore Deposition for Predicting Spread of Plant Diseases
}

\author{
Scott A. Isard and Marcelo Chamecki
}

First author: Department of Plant Pathology and Environmental Microbiology and Department of Meteorology, and second author: Department of Meteorology, The Pennsylvania State University, University Park 61802.

Accepted for publication 17 November 2015.

\begin{abstract}
Isard, S. A., and Chamecki, M. 2016. A physically based theoretical model of spore deposition for predicting spread of plant diseases. Phytopathology 106:244-253.

A physically based theory for predicting spore deposition downwind from an area source of inoculum is presented. The modeling framework is based on theories of turbulence dispersion in the atmospheric boundary layer and applies only to spores that escape from plant canopies. A "disease resistance" coefficient is introduced to convert the theoretical spore deposition model into a simple tool for predicting disease spread

of wheat leaf rust disease severity. The theoretical model has the advantage over empirical models in that it can be used to assess the influence of source distribution and geometry, spore characteristics, and meteorological conditions on spore deposition and disease spread. The modeling framework is refined to predict the detailed two-dimensional spatial pattern of disease spread from an infection focus. Accounting for the time variations of wind speed and direction in the refined modeling procedure improves predictions, especially near the inoculum source, and enables application of the theoretical modeling framework to field experiment design.
\end{abstract} at the field scale. Results from the model agree well with published measurements of Uromyces phaseoli spore deposition and measurements
Additional keywords: canopy escape, power law.
The spread of plant disease from infection foci depends on dispersal of spores from lesions to healthy, susceptible tissue (Gregory 1945). Dry dispersed spores (those not dispersed by precipitation) may be released into the air by some action of the organism or removed from lesions by wind (Ingold 1967). In the latter case, turbulence associated with eddies that penetrate downward into the plant canopy enhances removal of spores by disturbing the slower moving air layer immediately above lesions (Aylor 1999; Aylor and Parlange 1975). As airborne spores are advected downwind, turbulent eddies within the canopy are responsible for mixing them vertically. Spores released into a plant canopy have two possible fates: (i) some of them will be blown within the canopy until they are deposited on plant parts or the ground, generally close to the release site; and (ii) others may escape the canopy in upward moving eddies and travel for some distance in airflows before being deposited. The proportion of dry released spores that experience each of these fates depends on plant canopy structure and density, physical characteristics of the spores, spore release height, and turbulence (Aylor et al. 2001; Gleicher et al. 2014; Nathan et al. 2002; Pan et al. 2014).

When spore dispersal from an inoculum source occurs exclusively (or predominantly) within a plant canopy, the disease focus enlarges and spore dispersal and disease gradients at the boundary are very steep (Van der Plank 1975). In such cases, an exponential decay function has been employed to describe spore deposition

Corresponding author: M. Chamecki; E-mail address: chamecki@psu.edu

*The $\boldsymbol{e}$-Xtra logo stands for "electronic extra" and indicates that two supplementary files are published online.

http://dx.doi.org/10.1094/PHYTO-10-15-0275-R

(C) 2016 The American Phytopathological Society downwind from the focus (Frampton et al. 1942; Gregory and Read 1949):

$$
D(x)=a e^{-b x}
$$

where $D$ is the deposition flux, $x$ is distance from the focus, and $a$ and $b$ are empirically determined parameters. A function of the same form can be used to characterize disease intensity assuming that a fixed proportion of the deposited spores cause infections. Within canopy dispersal confines a pathogen to an overcrowded focus where further expansion can only occur along a boundary of ever-diminishing length in proportion to focus area (Van der Plank 1975). This pattern of dispersal and disease intensity is most prominent when spores are released into the lower portion of a dense canopy and/or are exposed to reduced levels of turbulence (Aylor 1978).

When turbulence is pronounced and/or release occurs high on plants, some of the spores are likely to escape into the airflow above the canopy. Generally in this situation, the spore deposition gradient downwind from the inoculum source is also steep (Gregory 1968, 1973; Van der Plank 1967). This is because many of the released spores deposit on nearby plant parts. In addition, spore escape is generally most pronounced immediately downwind from the release site and many of the spores that leave the canopy quickly return and deposit only a short distance downwind of their source lesion. In situations where a portion of the spores remain in the airflow above the canopy and are blown some distance downwind, the inverse power law, proposed by Gregory (1968) and modified by Mundt and Leonard (1985) provides a good description of the resulting pattern of spore deposition (Aylor 1987; Ferrandino and Aylor 1987; Sackett and Mundt 2005):

$$
D(x)=a(c+x)^{-b}
$$

where $a, b$, and $c$ are empirically determined parameters. Mundt (1989) suggests that $c$ is related to source area size while the 
theoretical framework for predicting $D(x)$ presented by Chamecki et al. (2012) attributes physical meaning to all the parameters in equation 2 . Because dispersal in airflows above a canopy may carry spores far and wide where they are likely to be deposited on nonhosts and/or where environmental conditions are less conducive for infection, the resulting pattern of disease intensity may differ from that of spore deposition.

Choosing a proper empirical model for describing spore deposition and the resulting disease gradient downwind from a source of inoculum can have important implications on interpreting field observations (Madden et al. 2007). The model selection process is necessarily constrained by the available data, being limited by the range of distances over which observations are obtained and measurement error. Different spore deposition models (equations 1 and 2) generally yield similar results for the region where data are available (usually $<10 \mathrm{~m}$ downwind) but produce very different results far from the source (Fig. 7.6 in Madden et al. 2007). Spore deposition models also yield contrasting patterns of disease epidemics. For example, describing observations of spore deposition with distance from an inoculum source using an exponential decay function corresponds to a disease wave that advances at a constant speed (Minogue and Fry 1983a, b; Van den Bosch et al. 1988), whereas using a power function to characterize spore deposition yields a dispersive disease front that accelerates (Ferrandino 1993; Minogue 1989; Mundt et al. 2009).

Empirical models for describing spore deposition are useful for explaining field data; however, they should be used with caution for making predictions about the spread of plant diseases beyond the domain of the observations. Coefficients in empirical models must be estimated from field observations using statistical procedures and thus vary from experiment to experiment. Even small variations in model coefficients can cause large differences in predictions of spore deposition and disease spread patterns (Madden et al. 2007). The objective of this manuscript is to present a physically based theory that overcomes this limitation and may be used to predict spore deposition downwind from an area source of inoculum for a variety of canopies. The theoretical framework was developed in Chamecki and Meneveau (2011) and Chamecki (2012) and used to explain observations of spore concentrations above a wheat field infected with leaf rust (Chamecki et al. 2012). It should be noted that the theory applies only to spores that escape from plant canopies (i.e., fate ii above) and thus have the potential to initiate new disease foci. It does not pertain to spores that exclusively travel and deposit within a canopy (i.e., fate $\mathrm{i}$ above), the primary agents of disease foci expansion. A "disease resistance" coefficient is introduced to convert the theoretical spore deposition model into a simple tool for predicting disease spread at the field scale.

Theory. Chamecki and Meneveau (2011) and Chamecki (2012) provide a theoretical modeling framework for predicting particle deposition from an area source. The model can be applied to the downwind deposition of spores that escape from a plant canopy with or without consideration of lateral dispersal. In rare situations where crosswind spread is not important because the maximum distance of spore deposition on susceptible hosts downwind is smaller than the inoculum source area, the solution is an inverse power law that can be recast in the form of equation 2 above (Chamecki and Meneveau 2011). In the more relevant case of inoculum dispersal from a small infected area within a large field or a landscape containing multiple susceptible host fields, deposition is a function of both downwind and crosswind directions and the solution to the theoretical model including lateral dispersal is not equivalent to that provided by the modified power law (Chamecki 2012).

Unlike empirical models, the theoretical framework for predicting spore deposition does not require fitting coefficients using observed data to determine the functional form of the dependence of spore deposition on distance from inoculum source. This is because the coefficients $a, b$, and $c$ in the solution to the theoretical model are calculated from information on spore properties (settling velocity $w_{s}$ ), meteorological conditions (friction velocity $\boldsymbol{u}_{*}$ ), and source characteristics (length of the inoculum producing area in the alongwind direction $L_{x}$, and average rate of spore release into the air or source strength $C_{0}$ ). More specifically,

$$
\begin{aligned}
& a=F_{T}(\beta-1)\left(\alpha \delta_{L}\right)^{\beta-1} \\
& b=\beta \\
& c=\alpha \delta_{L}
\end{aligned}
$$

In the theoretical framework, equations 2 to 5 are obtained from theories of atmospheric dispersion. However, the parameters $\alpha$ and $\beta$ cannot be predicted from the theory and must be determined from experiments. Chamecki and Meneveau (2011) and Pan et al. (2013) used a series of high-fidelity numerical simulations to obtain

$$
\begin{aligned}
& \alpha=8.0-2.75 \gamma \\
& \beta=1.08+1.25 \gamma
\end{aligned}
$$

Here, $\gamma$ is the Rouse number, a dimensionless measure of the relative importance of turbulence diffusion and gravitational settling, which is given by

$$
\gamma=\frac{\operatorname{Sc} w_{s}}{\kappa u_{*}}
$$

where Sc is the Schmidt number, the ratio between the eddy viscosity and eddy diffusivity for spores, and $\kappa$ is the von Karman constant. The derivation and assumptions of the theoretical model for predicting spore deposition are presented in Supplementary File S1. It should be noted, that the value of the exponent in equation 7 for the theoretical model, when applied to tracer particles (i.e., $\gamma=0$ ), differs from the power-law decay exponent $\beta=1.5$ obtained from Lagrangian stochastic models (Katul et al. 2005; Reynolds 2011). However, as discussed in detail by Pan et al. (2013), far away from the source, results obtained from equations 2 to 7 are in good agreement with those from rigorous theoretical studies on dispersion of heavy particles emitted from point sources obtained by Rounds (1955) and Godson (1958).

The effect of spore clumping during dispersal can be included in the model by adjusting $w_{s}$ to account for the abundance of clusters and number of spores per cluster (see Table 2 in Chamecki et al. 2012). Spore plume height $\left(\delta_{L}\right)$ and $F_{T}$, a measure of source strength, are quantities predicted by the model and characterize the inoculum source.

The height of the spore plume $\left(\delta_{L}\right)$ is given by

$$
\delta_{L}=\left[C_{1} \frac{\kappa z_{0}^{m}}{\operatorname{Sc} C_{p}}(m+1) L_{x}\right]^{1 /(\mathrm{m}+1)}
$$

Here, $C_{1}=(0.85 \mathrm{Sc} / \kappa-\gamma)$ is a constant, Sc is the turbulent Schmidt number (usually $\mathrm{Sc}=1$ is used), $\gamma$ is the Rouse number given by equation $8, z_{0}$ is the representative surface roughness length over the area source of spores, and $C_{p}=6$ and $m=1 / 7$ are constants describing the mean velocity profile in the neutrally stratified atmospheric surface layer (Brutsaert 1982). Calculating $F_{T}$ from the theory requires an estimate of $C_{0}$, the average concentration of spores above the canopy (see equation 13 in Chamecki 2012). The total flux of spores available for deposition immediately downwind from the source is given by $Q_{T}=F_{T} L_{y}$ (Chamecki 2012). Note that due to the difficulties in determining $C_{0}$, an alternative approach is to estimate $Q_{T}$ from the spore production and escape fraction and then calculate $F_{T}$ to use in equation 3. A third empirical approach to estimating $F_{T}$ is presented in the Material and Methods below.

For most applications, the inoculum source is small relative to the area that could potentially become infected, and thus spore 
deposition should be calculated using the theoretical model that accounts for spore dispersal in both downwind $(x)$ and crosswind $(y)$ directions (Chamecki 2012):

$$
D_{L}(x, y)=f(x, y) D(x)
$$

Here, $f(x, y)$ represents the effects of lateral dispersal. The model for $f(x, y)$ proposed by Chamecki (2012) is based on a Gaussian spread in the lateral direction integrated over the lateral extent of the source following the work for finite line sources pioneered by Sutton (1932). The final result is specified in terms of physical parameters

$$
f(x, y)=\frac{L_{y}}{2 L_{y}^{e q}}\left[\operatorname{erf}\left(\frac{y+L_{y}^{e q}}{\sqrt{2} \sigma_{y}^{e q}}\right)-\operatorname{erf}\left(\frac{y-L_{y}^{e q}}{\sqrt{2} \sigma_{y}^{e q}}\right)\right]
$$

where $L_{y}{ }^{e q}=L_{y}+\sigma_{y}\left(L_{x}\right)$ is the effective source size, $L_{y}$ is length of the source in the crosswind direction, and $\sigma_{y}(x)$ is the lateral plume spread, which can be modeled as $\sigma_{y}^{2}(x)=(1 / 2) C_{y}^{2} x p$. Following Sutton (1932), $C_{y}=0.225 \mathrm{~m}^{1 / 8}$ and $p=1.75$ are used to model the lateral dispersal of the plume. In equation 11, erf is the error function and $\sigma_{y}^{e q}(x)=\sigma_{y}\left(x+L_{x}\right)$ is the lateral plume spread evaluated at a distance $\left(x+L_{x}\right)$ from the source. Note that with the inclusion of lateral dispersal, the physically based model of spore deposition (equation 10) no longer follows a simple modified inverse power law (equation 2). However, this model preserves the power law decay along the centerline far from the inoculum source with an exponent,

$$
\beta_{\mathrm{eff}}=(\beta+p / 2)
$$

larger than that predicted by equation 7 (see equation 29 in Chamecki 2012). An extension of the theory that includes the effects of atmospheric stability on the deposition can be found in Pan et al. (2013). To account for atmospheric stability adjustments are made to the exponent of the power law (equation 7) and the height of the particle plume (equation 9). Including the effects of unstable atmospheric stratification in the theoretical model also requires atmospheric boundary layer height as an input parameter, a measurement seldom obtained during experimental campaigns.

The theoretical spore deposition model can be converted into a simple tool for predicting disease spread by incorporating terms that represent "disease potential" and "disease resistance." Disease potential $\phi(x, y)$ is defined as the maximum possible level of disease at a given location under ideal conditions as a consequence of spore deposition. It must be noted that the theory applies only to spores that escape from plant canopies (i.e., fate ii above) and deposit on susceptible hosts downwind. In the theoretical model with lateral dispersal included, this potential is set by spore availability which is equivalent to spore deposition, $\phi_{L}(x, y)=D_{L}(x, y)$. It is unlikely that this potential is ever realized in nature. Consequently, a "disease resistance" term $0 \leq R(x, y) \leq 1$ is introduced that limits the appearance of disease symptoms based on characteristics of the deposited spores (viability), host (susceptibility and the availability of healthy tissue), and environmental conditions (temperature and moisture). In this framework, the severity of disease as a consequence of the spore deposition is given by the theoretical model with and without accounting for lateral dispersion, respectively, by

$$
S_{L}(x, y)=(1-R(x, y)) \phi_{L}(x, y)=(1-R(x, y)) D_{L}(x, y)
$$

and

$$
S_{\infty}(x, y)=(1-R(x, y)) \phi_{\infty}(x, y)=(1-R(x, y)) D_{\infty}(x, y)
$$

Here and below, the subscript $\infty$ is used to bring attention to the equivalence between ignoring crosswind spore dispersal and the assumption that the inoculum source is infinite in the crosswind direction.

\section{MATERIALS AND METHODS}

Modeling framework. Two forms of the physically based theoretical model are employed; a simple approach for predicting spore deposition and disease spread downwind from an inoculum source and a more refined procedure for predicting the detailed twodimensional spatial pattern of disease spread from an infection focus. For validation purposes, values of spore deposition and lesion counts per leaf at distances downwind from the edge of the inoculum source are calculated using the theoretical model with and without lateral dispersal included. These predictions and the observations are log-transformed (using the natural base) and coefficients of determination ( $R^{2}$ values) are calculated following Sackett and Mundt (2005) (Table 1) for comparison with statistics from previously published studies using empirical deposition models. In the sections below, $x$ is distance measured from the downwind edge of the inoculum source.

Simple theoretical modeling approach for predicting spore deposition and disease spread downwind from an inoculum source. To predict spore deposition in the downwind direction using equation 10, five parameters are required: length and width of the inoculum source area, settling velocity for spores, friction velocity, and a measure of source strength. The settling velocity for spores can be measured using simple laboratory equipment (Chamecki et al. 2011; Di-Giovanni et al. 1995; Ferrandino and Aylor 1984; Loubet et al. 2007) or estimated from spore diameter $d_{p}$ and density $\rho_{p}$, using Stokes law (Gregory 1973):

$$
w_{s}=\frac{\rho_{p} d_{p}^{2} g}{18 \mu}
$$

Here, $g$ is the gravitational acceleration and $\mu$ is the dynamic viscosity of air.

TABLE 1. List of model parameters used for each experiment ${ }^{\mathrm{a}}$

\begin{tabular}{lccccccccccc}
\hline Experiment & $u_{*}(\mathrm{~m} / \mathrm{s})$ & $w_{s}(\mathrm{~m} / \mathrm{s})$ & $L_{x}(\mathrm{~m})$ & $L_{y}(\mathrm{~m})$ & $h(\mathrm{~m})$ & $z_{0}(\mathrm{~m})$ & $\gamma$ & $\beta$ & $\beta_{\text {eff }}$ & $c(\mathrm{~m})$ & $R^{2}$ \\
\hline Bean field & & & & & & & & & & & \\
Singlets & 0.33 & 0.01 & 1.00 & 1.00 & 0.3 & 0.038 & 0.08 & 1.18 & 2.05 & 1.01 & 0.91 \\
Clusters of 5 & 0.33 & 0.02 & 1.00 & 1.00 & 0.3 & 0.038 & 0.19 & 1.32 & 2.19 & 0.92 & \\
Wheat field $^{\mathrm{c}}$ & 0.51 & 0.04 & 1.52 & 1.52 & 1.0 & 0.125 & 0.20 & 1.33 & 2.20 & 1.55 & 0.97 \\
Wheat field $^{\mathrm{d}}$ & 0.33 & 0.04 & 1.52 & 1.52 & 1.0 & 0.125 & 0.30 & 1.46 & 2.34 & 1.43 & 0.81 \\
Wheat field $^{\mathrm{e}}$ & 0.42 & 0.04 & 1.52 & 1.52 & 1.0 & 0.125 & 0.24 & 1.38 & 2.26 & 1.47 & 0.96 \\
\hline
\end{tabular}

a Symbols in table represent friction velocity $\left(u_{*}\right)$, spore settling velocity $\left(w_{s}\right)$, length of the inoculum producing area in the along-wind $\left(L_{x}\right)$ and crosswind $\left(L_{y}\right)$ directions, height of the underlying canopy $(h)$, surface roughness $\left(z_{0}\right)$, Rouse number $(\gamma)$, power law exponent $(\beta)$, effective power law exponent $\left(\beta_{\text {eff }}\right)$, model parameter related to source area size $(c)$, and coefficient of determination $\left(R^{2}\right)$.

b Ferrandino and Aylor (1987) Experiment 2.

c Sackett and Mundt (2005) Hermiston 2002.

d Sackett and Mundt (2005) Madras 2002.

e Sackett and Mundt (2005) Madras 2003. 
Friction velocity can be estimated using eddy-covariance techniques or measurements of mean wind speed using the logarithmic law-of-the-wall for the mean wind speed profile:

$$
\bar{u}(z)=\frac{u_{*}}{\kappa} \ln \left(\frac{\left(z-d_{0}\right)}{z_{0}}\right)
$$

In equation $16, \kappa=0.4$ is the von Karman constant, $\bar{u}(z)$ is mean wind speed, $z$ is height of wind speed measurement, $d_{0}$ is displacement height, and $z_{0}$ is surface roughness. Values of $d_{0}$ and $z_{0}$ can be estimated using $d_{0}=2 h / 3$ and $z_{0}=h / 8$, where $h$ is the height of the underlying canopy (Brutsaert 1982).

As noted above, $F_{T}$ can be considered a measure of source strength, a parameter that is difficult to quantify. When assessing model predictions by comparison with observations, determining source strength may be avoided by normalizing the data set using spore deposition measurements from an appropriate location at the downwind edge of the source (Ferrandino and Aylor 1987, see below). This procedure assumes that the error associated with this single deposition measurement is minimal. An alternative common approach employed here is to statistically determine $F_{T}$, using equations 2 or 10 , so that the mean squared error between model predictions and data for all downwind measurement locations is minimized. It should be noted that this procedure assumes that the measurement errors across the multiple locations employed in the calculations are randomly distributed. It does not affect the shape of the curve predicted by the theoretical model (e.g., the shape of the curve depicted on a plot of deposition [ordinate] versus distance [abscissa]); it simply impacts model predictions by displacing the curve by a vertical offset that minimizes the deviations from observations.

To predict disease spread in the downwind direction using equations 13 and 14, the same five parameters are required: length and width of the inoculum source area, settling velocity for spores, friction velocity and a measure of source strength. Here the disease resistance term, $R(x, y)$, is treated as an unknown constant. Consequently, the product $R F_{T}$ is statistically determined so that the mean squared error between model predictions and disease severity determinations for all downwind measurement locations is minimized.

Refined theoretical modeling procedure for predicting the detailed two-dimensional spatial pattern of disease spread from an infection focus. The simple theoretical modeling approach described above can be refined to predict the detailed twodimensional spatial pattern of disease spread from an infection focus resulting from a long period of spore dispersal (e.g., days to weeks). In this procedure, friction velocity is calculated from hourly wind measurements throughout the study period. A fine numerical grid is established with the inoculum source at its center. The theoretical model including lateral dispersion (equation 13) is then applied using the mean wind direction for each hour to project the spores deposited from the plume onto the numerical grid. The resulting deposition fields for each hour are added and $R F_{T}$ is estimated as described above. Once again, the determination of $R F_{T}$ only modifies the predictions throughout the domain by a constant factor and does not impact the two-dimensional patterns predicted by the theoretical model.

Validation data sets. Data sets appropriate for validating physically based models of aerial dispersal of pathogens and the diseases they cause are rare, and never truly ideal. As indicated, validation of models for predicting spore dispersal requires deposition measurements at multiple locations extending far from the source (e.g., $>10 \mathrm{~m}$ ). Because spore deposition gradients are very steep, relatively long periods with minimal variation in wind direction are necessary to capture large enough quantities of spores at distant measurement locations for meaningful model validation. Creating data sets appropriate for validating models of disease spread due to aerial spore dispersal is even more challenging, requiring assessments at multiple locations of disease severity associated with second generation lesions produced from an isolated infection focus. Environmental conditions favorable for infection must be maintained throughout a large field planted to a susceptible host. In addition, wind speed and direction must be monitored during the measurement period, the length of which is determined by pathogen generation time. The field studies described below reported in Ferrandino and Aylor (1987) and Sackett and Mundt (2005) meet the stringent requirements for validating predictive models of spore deposition and disease spread. To further demonstrate the robustness of the theoretical modeling framework and provide additional insights for model application to spore dispersal, comparisons of results using equations 2 and 10 with the extensive observational data set of pollen deposition reported by Raynor et al. (1970) are provided in Supplementary File S2.

Spore deposition within a bean field. Ferrandino and Aylor (1987) measured deposition of urediniospores of Uromyces phaseoli, the causal agent of bean rust, released from a line source in a snap bean field (Phaseoli vulgaris). Inoculum source areas were established $30 \mathrm{~m}$ apart in each of the quadrants of a rectangular field and deposition from only the upwind source was measured in each experiment to avoid possible interference. The source areas consisted of $1 \mathrm{~m}$ lengths of two contiguous bean rows. Deposition was assessed by enumerating urediniospores on sticky microscope slides positioned on the ground and at $0.25 \mathrm{~m}$ heights along arcs with $1,2.5,4$, and $5 \mathrm{~m}$ radii downwind from the center of the source. In addition, 20 to 25 potted bean plants were used to trap spores and lesions on these plants were counted after an incubation period. The trap plants were positioned at approximately the same height as the elevated slides and were located $3.8 \mathrm{~m}$ apart in a square grid extending $16 \mathrm{~m}$ downwind from the center of the inoculum source. Experimental runs were conducted once numerous sporulating pustules developed on the source plants (12 to 14 days after inoculation) and lasted $4 \mathrm{~h}$ (0900 to $1300 \mathrm{~h} \mathrm{EST})$. Data from the elevated slides and trap plants were combined. Results are reported as a function of distance from the center of the infected area and are normalized by values measured $1 \mathrm{~m}$ downwind. Data for single spores deposited between 2 to $15 \mathrm{~m}$ downwind of the source obtained from the second experiment are reported by the authors (Fig. 3 in Ferrandino and Aylor 1987) and are used here. Equation 10 is parameterized for this experiment using: length $(1 \mathrm{~m})$ and width $(1 \mathrm{~m})$ of the inoculum source area, settling velocity for urediniospores of $U$. phaseoli $(0.01 \mathrm{~m} / \mathrm{s}$ from Ferrandino and Aylor 1984), friction velocity $(0.33 \mathrm{~m} / \mathrm{s}$ from Table 1 in Ferrandino and Aylor 1987), and a measure of source strength. The information provided by the authors does not enable estimation of $F_{T}$. Thus, $F_{T}$ is determined as explained above, so that the mean squared error between model predictions and experimental data are minimized (only data for locations where $x>1 \mathrm{~m}$ are used).

Disease severity within a wheat field. Sackett and Mundt (2005) present measurements of primary disease gradients of wheat stripe rust caused by Puccinia striiformis within three replicated plots at two locations (Madras and Hermiston, OR) for 2 years. The plots planted to winter wheat 'Jarvis' susceptible to $P$. striiformis were $6.1 \mathrm{~m}$ wide by 128 to $171 \mathrm{~m}$ long oriented in the direction of the dominant wind (west to east) and separated by buffer zones (16.8 to $30.5 \mathrm{~m}$ wide) planted to a resistant winter wheat cultivar. Inoculum source areas $(1.52 \times 1.52 \mathrm{~m})$ were established in the spring of each year in the upwind portion of each plot. Within each experiment, foci received equal amounts of inoculum. The inoculated plants were covered overnight to prevent the spread of spores beyond the intended area and to enhance environmental moisture for germination and infection. Starting in the spring, irrigation was applied once and three to five times each week at Madras and Hermiston respectively. Intensive disease assessments were conducted in each plot shortly after the appearance of the second generation of the pathogen and before the appearance of the third generation. Disease severity along the centerline of the plot was 
measured at distances of $0.9,1.5,2.1,3.0,4.6,6.1,9.1$, and $12.2 \mathrm{~m}$ from the center of the focus and every $6.1 \mathrm{~m}$ thereafter in both the upwind and downwind directions. Initial disease establishment at the Hermiston site in 2003 was weak due to dry conditions after inoculation and consequently only the three largest downwind data sets (labeled Hermiston 2002, Madras 2002, and Madras 2003) are used to evaluate the theoretical modeling framework.

Friction velocity was estimated based on meteorological data available from the AgriMet website (www.usbr.gov/pn/agrimet/) for stations located close to the experimental sites. Average wind speeds were calculated over the dispersal period (corresponding to the second latent period assumed to last 21 days, see Sackett and Mundt 2005). Only values when the wind direction was from the west $\left( \pm 45^{\circ}\right)$ were used in the simple theoretical modeling approach, resulting in mean wind speeds at $3 \mathrm{~m}$ above the ground equal to 4.0, 2.6, and $3.3 \mathrm{~m} / \mathrm{s}$ for Hermiston 2002 and Madras 2002 and 2003 , respectively. Values of friction velocity obtained from these mean wind speed data and the settling velocity for $P$. striiformis urediniospores are shown in Table 1.

Because Sackett and Mundt (2005) report disease severity, "disease resistance" is incorporated in the theoretical model (equations 13 and 14) to evaluate predictions. It is assumed that both host susceptibility and environmental conditions are uniform throughout each experimental plot so that $R$ is constant and consequently, disease severity is proportional to spore deposition. In this experiment source strength $\left(F_{T}\right)$ is also unknown, so the constant $R F_{T}$ is determined from disease severity observations as described above (except that here only data for locations where $x>5 \mathrm{~m}$ is used).

\section{RESULTS}

Simple theoretical modeling approach. Comparisons between model predictions and measurements are shown in Figures 1 and 2 for the Ferrandino and Aylor (1987) and Sackett and Mundt (2005) field experiments, respectively. A list of the input parameters, key predicted quantities, and coefficients of determination for model simulations of the experimental data sets are shown in Table 1.

Theoretical model predictions are shown with and without consideration of lateral dispersion $\left(D_{L}\right.$ from equation 10 and $D_{\infty}$ from equation 2 with parameters calculated from equations 3 to 7 , respectively, in Figure 1 and $S_{L}$ and $S_{\infty}$ from equations 13 and 14, respectively, in Figure 2). A close inspection of the panels in both figures reveals that the inverse power law model predictions which neglect lateral dispersal (dash lines) do not capture the correct decay of deposition and disease severity with distance as well as the theoretical model that accounts for lateral dispersal (solid lines). This is due to the small size of the inoculum source areas $(1 \times 1 \mathrm{~m}$ and $1.52 \times 1.52 \mathrm{~m}$ for the two field experiments, respectively). As discussed above, lateral dispersal has a pronounced impact on downwind spore deposition gradients when the crosswind dimension of a source area is small relative to the downwind dispersal distance.

Predictions of deposition for single spores using the simple theoretical modeling approach with lateral dispersion (equation 10) are in very good agreement $\left(R^{2}=0.91\right)$ with data sets for elevated slides and trap plants shown in Figure 1A. Note that these $R^{2}$ values are independent of the statistically determined parameter (multiplicative constant $F_{T}$ ) in the theoretical model and thus reflect the predictive capabilities of the model. The effect of clumping on model predictions of normalized spore deposition can also be estimated using equations 2 or 10 . In essence, the larger settling velocity of clumps results in a larger exponent for the power-law decay. As an example, the model given by equations 2 to 7 predicts $\beta=1.18$ for single spores and $\beta=1.32$ for clumps of five spores for the bean rust (Table 1). Ferrandino and Aylor (1987) report $\beta=2.4 \pm 0.5$ and $\beta=2.2 \pm 0.4$ using the empirical modified power law model for elevated slides and potted plants for singlets and clusters of five or more spores respectively in their experimental run 2 , and $\beta=2.1 \pm 0.3$ for the three experimental runs combined. The theoretical model that includes lateral dispersal predicts $\beta_{\text {eff }}=2.05$ and $\beta_{\text {eff }}=2.19$ for singlets and clusters of five spores respectively for run 2 , values within the error measures associated with the empirically modeled parameters. Results for the ground deposition slides are shown in Figure 1B for run 2. The decay of deposition with distance is less dramatic than for the elevated surfaces (Fig. 1A). Clearly this data set includes spores that have dispersed exclusively within the canopy (fate i) in addition to those that have escaped the canopy before reentering the canopy and depositing on the ground (fate ii). The empirically determined power law model (equation 2) appears to better capture the decrease in spore deposition with distance than the empirical exponential decay function (equation 1) as well as the theoretical model with and without lateral dispersal (equation 10 and equation 2 with parameters calculated from equations 3 to 7 , respectively). Unfortunately the deposition measurements are limited to distances too close to the source $(\leq 5 \mathrm{~m})$ to afford confidence in the empirically estimated model parameters. Despite the slight under predictions of deposition near the source, it is clear that the theoretical model which includes lateral dispersal captures the decay of ground deposition with distance characteristic of this region.
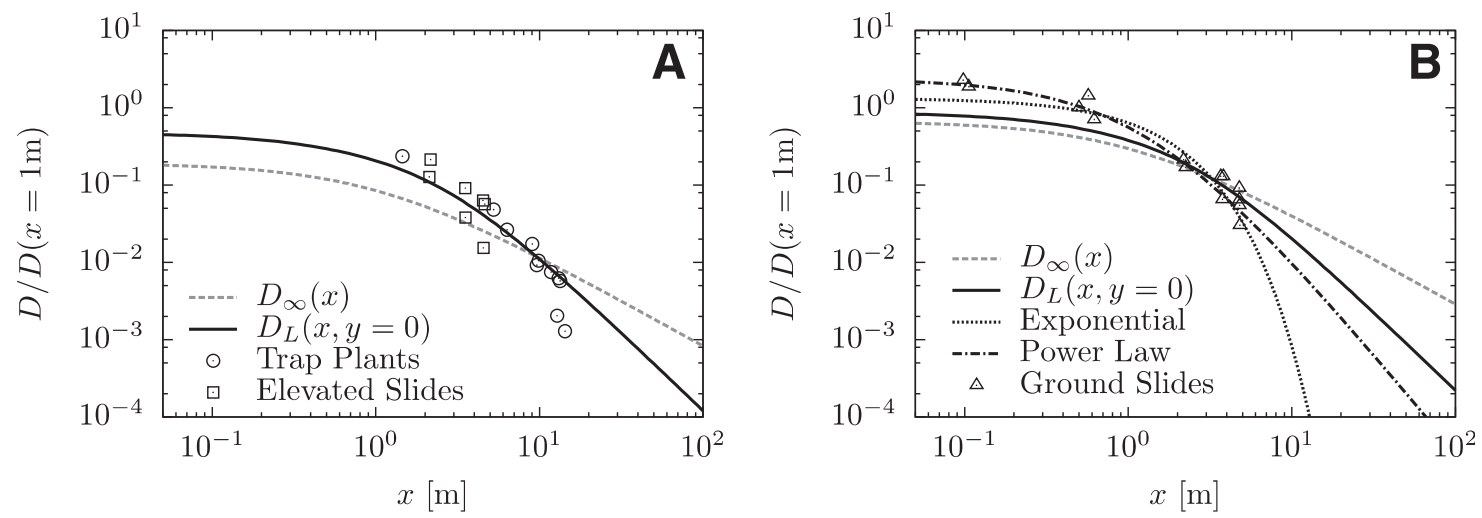

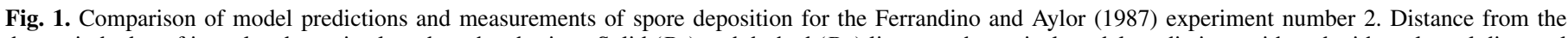

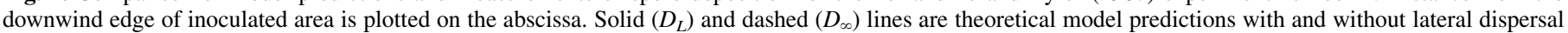

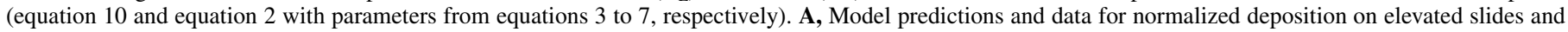

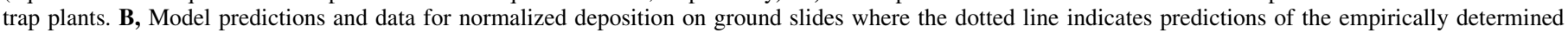

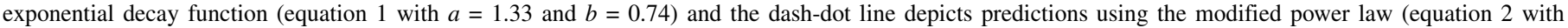
parameters $a=4.75, b=2.56$, and $c=1.31$ empirically determined). 
Comparisons between theoretical model predictions with and without lateral dispersal $\left(S_{L}\right.$ from equation 13 and $S_{\infty}$ from equation 14 , respectively) and measurements of disease severity for the three data sets provided by Sackett and Mundt (2005) are presented in Figure 2. Agreement between the predictions of the model including lateral dispersal (solid line) and experimental data are
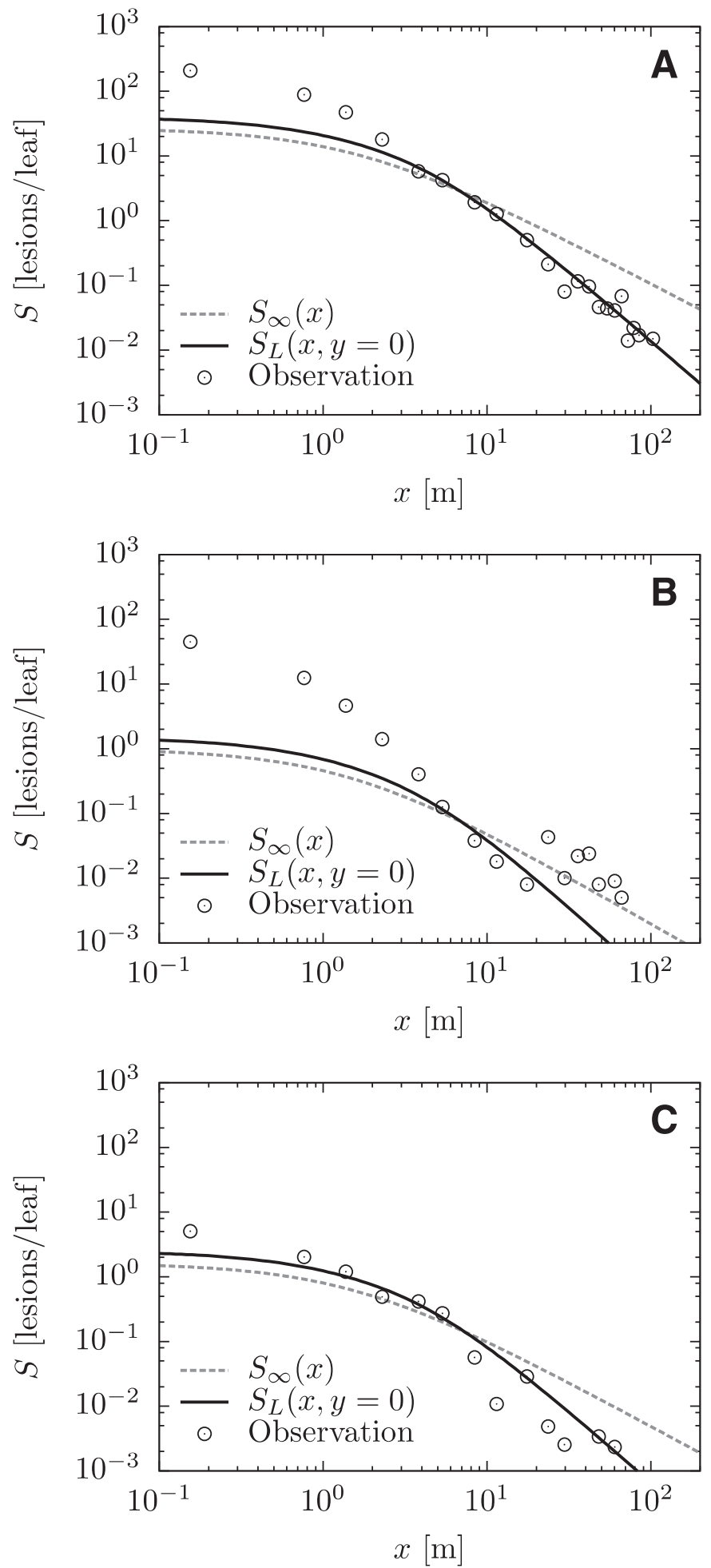

Fig. 2. Comparison of model predictions and measurements of disease severity for the three largest downwind data sets (A, Hermiston 2002, B, Madras 2002, and C, Madras 2003) presented in Sackett and Mundt (2005). Distance from the downwind edge of inoculated area is plotted on the abscissa. Solid $\left(S_{L}\right)$ and dashed $\left(S_{\infty}\right)$ lines are theoretical model predictions for the downwind direction with and without lateral dispersal (equations 13 and 14, respectively). Open circles are means of disease severity measurements from two or three replicated plots. excellent for the Hermiston 2002 data set $\left(R^{2}=0.97\right)$ shown in panel Figure 2A; the only exception being for disease severity very close to the edge of the inoculum source area $(x<3 \mathrm{~m})$. Very good agreement $\left(R^{2}=0.96\right)$ is also obtained for Madras 2003, shown in Figure $2 \mathrm{C}$. In this case, equation 13 clearly captures the transition between the zone of nearly constant disease severity, near the inoculum source, to the larger downwind section of the field where the disease gradient is well characterized by the modified power law model with physically based parameters (dash line is parallel to pattern made by observations $>10 \mathrm{~m}$ from source). Even though results of equation 13 are not as good for the Madras 2002 data set $\left(R^{2}=0.81\right)$ shown in Figure $2 \mathrm{~B}$, the theoretical model including lateral dispersal appears to capture the decrease in disease severity with distance for locations more than a few meters from the source. As pointed out by Sackett and Mundt (2005), in this case "winds during the dispersal period were not from the expected direction, [and] data suggested that the observed gradients may have been influenced by inoculum from other plots in the field."

Insight to the effect of wind speed variability during the dispersal period (second latent period lasting 21 days) on the results from the simple theoretical modeling approach including lateral dispersal (equation 13) is provided in Figure 3. The Hermiston 2002 data set is used for the analysis because the winds were stronger and more consistent and disease severity during the first generation was greater in the inoculation source area than for the other experiments. Figure 3 shows results from equation 13 using friction velocity calculated from the mean wind speed as well as values for $u^{*}$ that are both $1.2 \sigma_{u} *$ larger and smaller than the mean (here $\sigma_{u *}$ is the standard deviation of the hourly values of $u^{*}$ during the 21 days). It is apparent from Figure 3 that the disease observations near the inoculum source are predicted well by equation 13 using the lower value of wind speed while the decrease in disease severity with distance beyond a few meters downwind of the source is well predicted using the higher value of wind speed in the model. Clearly, periods of low wind speed should result in increased deposition near the inoculum source while spores are likely to be carried to more distant locations by winds with higher velocities. Thus, it is possible that wind speed variability may explain the under prediction of disease severity near the inoculum source calculated for Hermiston 2002 using the theoretical model that includes lateral dispersal. This speculation led to the refined two-dimensional modeling procedure and the results that follow.

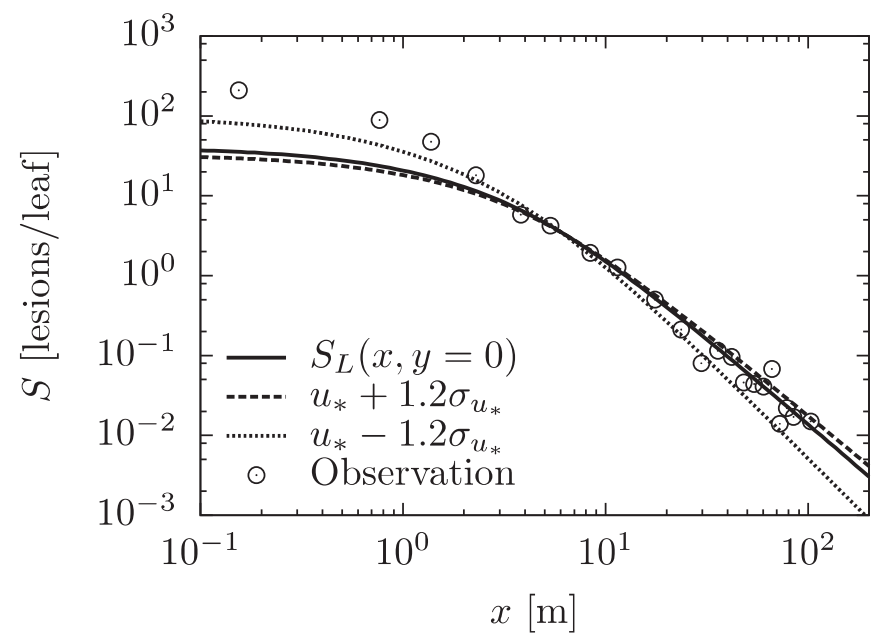

Fig. 3. Sensitivity of disease severity predictions from the theoretical model including lateral dispersal (equation 13) to wind speed, represented in the model by the value of the friction velocity $u_{*}$. Distance from the downwind edge of inoculated area is plotted on the abscissa. Circles represent disease severity observations from Hermiston 2002 experiment. Dashed and dotted lines represent predictions using equation 13 in which the values for $u^{*}$ are increase and decrease by $1.2 \sigma_{0^{*}}$. 
Refined theoretical modeling procedure. Disease severity predictions using the refined modeling procedure for the center portion of the large numerical grid are shown in Figure 4. The solid line depicts the experimental plot (mean dimensions of the two replicate plots). The small white region in and around the inoculum source delineates the area where the theoretical model that includes lateral dispersal cannot be applied without taking into consideration spores dispersed within the canopy (fate i). The direction to the right of the source is east and is considered downwind in the analysis. A close inspection reveals that disease severity is most intense in the downwind direction (most of the purple area surrounding the source lies to the right of the plot origin) but that disease severity is also pronounced outside the plot in other directions (see dash contours in Figure 4). The full spatial representation of spore deposition and disease spread from an inoculum source generated by the refined

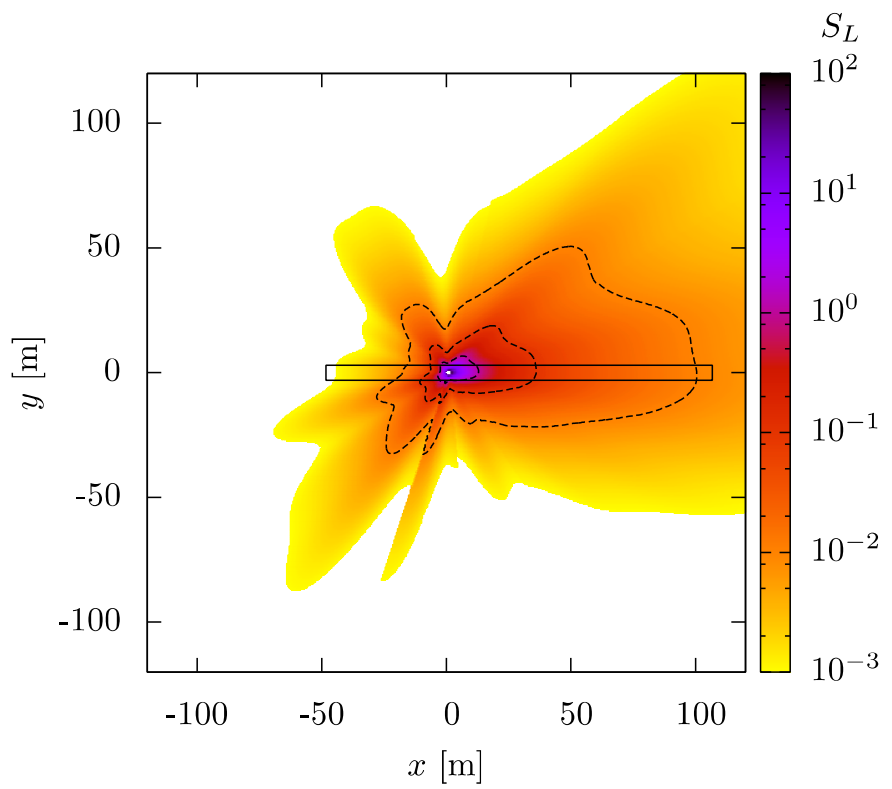

Fig. 4. Disease severity predictions using the theoretical model including lateral dispersal (equation 13) in the detailed modeling approach. Only the center portion $(120 \times 120 \mathrm{~m})$ of the larger numerical grid is displayed. The solid line depicts the experimental plot (mean dimensions of the two replicate plots). The small white area within the plot represents the region in and around the inoculum source and delineates the area where the model cannot be applied without taking into consideration spores dispersed within the canopy. The direction to the right of the source is east and is considered downwind in Sackett and Mundt (2005). The dash lines represent 1.0, 0.1, and 0.01 lesions/leaf contours. modeling procedure provides a practical tool for researchers. In this case, model results confirm the claim that interplot contamination was unlikely during this experiment since the two replicate plots were parallel and $62.4 \mathrm{~m}$ apart (Sackett and Mundt 2005) (Fig. 1) and infection in the intervening plots was very low on the measurement date (Cowger et al. 2005). A comparison of disease severity $\left(S_{L}\right)$ predictions from the simple theoretical modeling approach and refined procedure with field observations for the Hermiston 2002 experiment is displayed in Figure 5. Agreement between predictions using the refined modeling procedure and experimental data for the downwind measurements is excellent $\left(R^{2}=0.99\right)$, matching the observations close to the source better than the predictions from the simple modeling approach (Fig. 5A). In addition, the agreement for the upwind measurements (Fig. 5B) is only slightly less $\left(R^{2}=0.97\right)$, which is particularly satisfying since $R F_{T}$ was determined based only on the observations from the eastern side (downwind) of the source. It should be noted that the modified power law model coefficients $a, b$, and $c$ differ when empirically estimated using disease observations from the west (upwind) and east (downwind) sides of the inoculum source (Sackett and Mundt 2005) (Table 1). The differences can be attributed to the anisotropy of key physical parameters (in this case, wind speed and direction). Consequently, the empirical modified power law model should only be used to make inferences about disease spread in the direction for which the field observations used to determine the parameters are obtained.

\section{DISCUSSION}

Spore deposition gradients play an important role in the development of aerially propagated plant disease epidemics. Unfortunately, these gradients are steep and thus experimental data rarely extend far enough from the inoculum source to enable one to confidently choose between different empirical deposition gradient models. In exceptional studies, Ferrandino and Aylor (1987) and Sackett and Mundt (2005) provide convincing observational evidence that a modified power law is the best empirical approach. The work of Chamecki and Meneveau (2011) and Chamecki (2012) provides a framework anchored on the theories of turbulence dispersion in the atmospheric boundary layer to model spore deposition. Chamecki and Meneveau (2011) showed that the modified power law first proposed by Mundt and Leonard (1985) is the appropriate functional form to describe deposition patterns if crosswind dispersion is not important. Inclusion of crosswind dispersion by Chamecki (2012) leads to an improved model that preserves the power law decay far from the inoculum source with an increased exponent $\beta_{\text {eff. }}$ For the case studies presented here, a simple modeling approach using the theoretical model accounting
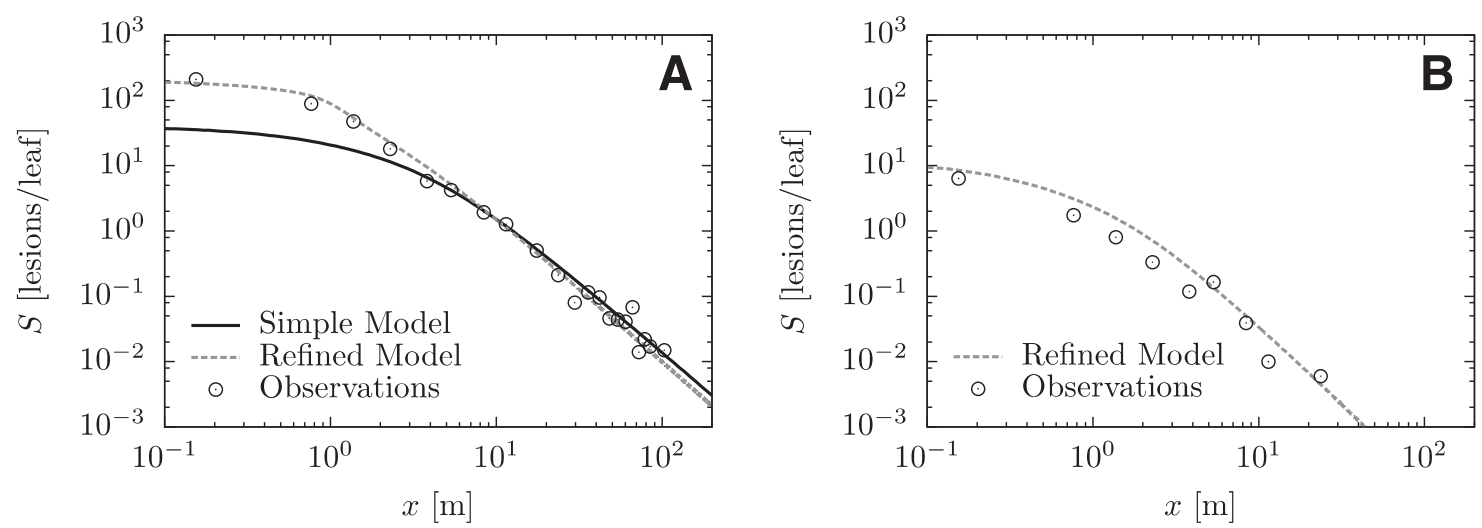

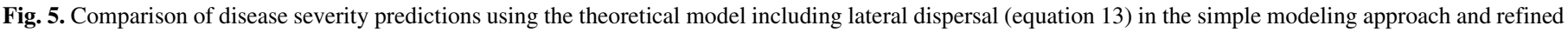

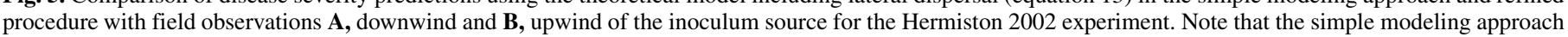

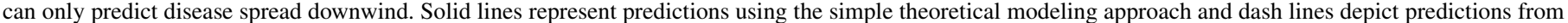
the refine modeling procedure. Open circles are means of disease severity measurements from two replicated plots. 
for crosswind dispersal produces accurate predictions of spore deposition in the downwind direction, especially for distances larger than about $3 L_{y}$ from the source. To support this claim in light of the dearth of data sets appropriate for validating physically based models of aerial dispersion of pathogens, comparisons of theoretical model results with observational data of pollen deposition are presented in Supplementary File S2. The expansion of the validation data sets to include larger particles reveals that the theoretical model with lateral dispersal performs well over a wide range of $\gamma(0.08 \leq \gamma \leq 1.28)$ and is equally applicable to predicting particle deposition downwind from small and large area sources. The resulting validated theoretical model provides researchers with a practical tool for predicting spore deposition and disease spread with distance from an area source of inoculum.

It is important to emphasize that model predictions presented here are "true predictions" of spore deposition and disease severity gradients (requiring determination of the source strength $F_{T}$ or the composite constant $R F_{T}$ ). Comparisons between observations and empirical model predictions such as those reported here and in empirical studies such as Ferrandino and Aylor (1987) and Sackett and Mundt (2005) must be interpreted with care. Empirical models with three degrees of freedom (three constants must be estimated from observations to apply the modified inverse power law) fit to field data are likely to produce very good agreement with observations, but carry no capability to predict spore deposition and disease spread for conditions outside those encountered during data collection. In contrast, the theoretical model requires determination of a single constant from field observations, which does not impact the prediction of rate of decrease in spore deposition and disease spread with distance from the inoculum source. Consequently, the theoretical model is widely applicable for studies involving spore deposition and disease spread (under ideal conditions) requiring only five parameters, length and width of the inoculum source area, spore settling velocity, friction velocity, and an appropriate measure of source strength.

In addition to the model validation aspect of the present work, a relationship between spore deposition and disease severity is proposed in which environmental conditions act to reduce the conversion of deposition ("disease potential") into realized infection (defining a coefficient of "disease resistance"). For near ideal conditions, such as the experimental data sets used here, the coefficient $R(x, y)$ for the irrigated fields planted to a highly susceptible cultivar can be assumed to be constant (i.e., not varying in space). By estimating the value of the multiplicative parameter $R F_{T}$, so that the mean squared error between model predictions and experimental data are minimized, the theoretical model that includes lateral dispersal provides accurate predictions of the disease severity observed in these experiments. The predictions of downwind decay of disease severity for distances larger than $10 L_{x}$ from the source using the theoretical model display a power law form supporting observational evidence that focal epidemics of wheat stripe rust expand as dispersive waves (Cowger et al. 2005).

Even though predictions of the physically based theory that includes lateral dispersal of spores proposed here display a power law form at distances larger than $10 L_{x}$ from the source, the model yields a solution that is not equivalent to the modified power law. Nevertheless in most cases, there should be a high correspondence between the values of the $\beta_{\text {eff }}$ coefficient in the theoretical model with lateral dispersal and $b$ in the modified power law (determined either empirically from equation 2 or theoretically from equation 7 and 12 with a typical value of 1.75 for $p$ ). Because the modified power law with empirically determined parameters also closely approximates field observations, it remains a useful empirical tool for estimating spore deposition and disease spread in the downwind direction where field observations are available from multiple locations extending far enough from the source to accurately determine model parameters. Additional assumptions regarding spatial homogeneity of host susceptibility and environmental conditions are required for the application of the theoretical model to disease spread.

The theoretical model with or without lateral dispersal has the advantage over empirical models in that it can be used to assess the
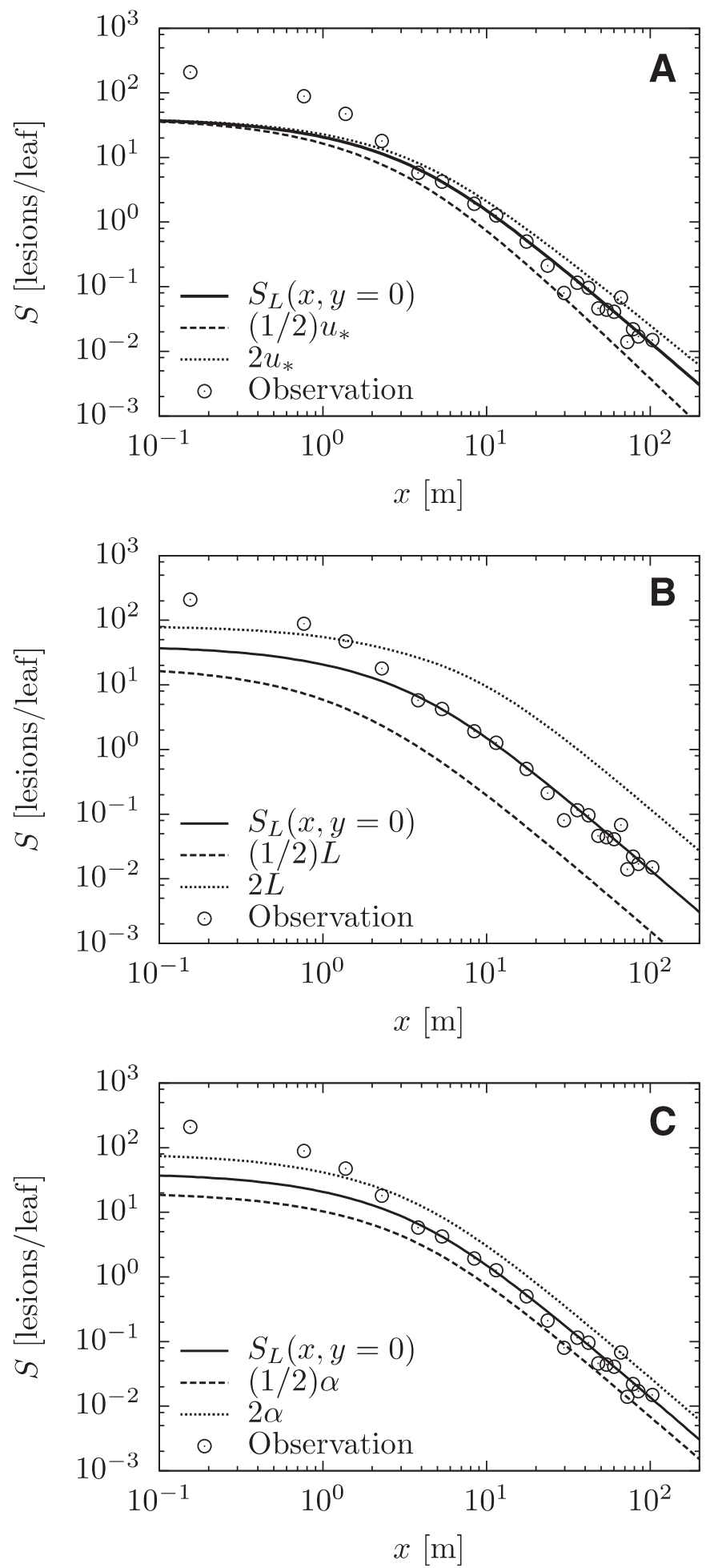

Fig. 6. Disease spread in the downwind direction using the theoretical model including lateral dispersion (equation 13) in the simple modeling approach and the Hermiston 2002 data set. Sensitivity to changes in A, mean wind speed, B, source size, and $\mathbf{C}$, aspect ratio of the source area are displayed. The circles represent disease observations, solid lines represent model prediction using observed experimental conditions, and the dot lines are model predictions obtained by multiplying and dividing the mean wind speed, source size, and aspect ratio by two. It should be noted that changes in source size alter source strength according to the model equations. 
influence of source distribution and geometry, spore characteristics, and meteorological conditions on spore deposition and disease spread. Figure 6 shows the sensitivity of disease spread in the downwind direction to changes in mean wind speed (Fig. 6A), source size (Fig. 6B), and aspect ratio of the source (Fig. 6C) using the theoretical model including lateral spread (equation 13) in the simple modeling approach and the Hermiston 2002 data set. The circles represent disease observations, solid lines represent model predictions using observed experimental conditions and the shaded areas are delineated by model predictions (dot lines) obtained by multiplying and dividing the mean wind speed, source size, and aspect ratio by two. It should be noted that changes in source size alter source strength according to the model equations. The analysis provides insight into the effect of the individual variables on disease spread and the magnitude of the error that might be incurred when using an empirical model under conditions different from those for which parameters were estimated. It also reveals the high relative importance of inoculum source size on disease spread. In addition, results presented in Figure 6 illustrate the biases in predictions that would be incurred if the empirical model were applied outside the conditions under which the constants $a, b$, and $c$ were fitted to observations. As an example, assume that the solid line in Figure 6A was obtained by empirically fitting $a, b$, and $c$ in the modified power law model to disease severity (or spore deposition) field data. Then suppose that the same coefficients were used in the empirical modified power law model to predict disease under different circumstances (e.g., subsequent year, different location, or another infection cycle), during similar conditions except that wind speeds were $50 \%$ lower than during the initial measurement period (i.e., equivalent to the dot line in Figure 6A). In that case, the expected disease severity $100 \mathrm{~m}$ downwind from the source predicted by the empirical model would be approximately 1 order of magnitude greater than the theoretical model prediction.

Accounting for the time variations of wind speed and direction in the refined theoretical modeling procedure enables prediction of the detailed two-dimensional spatial pattern of disease spread from an infection focus. The refined procedure also improves predictions of disease severity, especially near the inoculum source, and enables application of the theoretical model that includes lateral spread to field experiment design. Where experiments using aerially dispersed plant pathogens are replicated in nearby locations (e.g.,

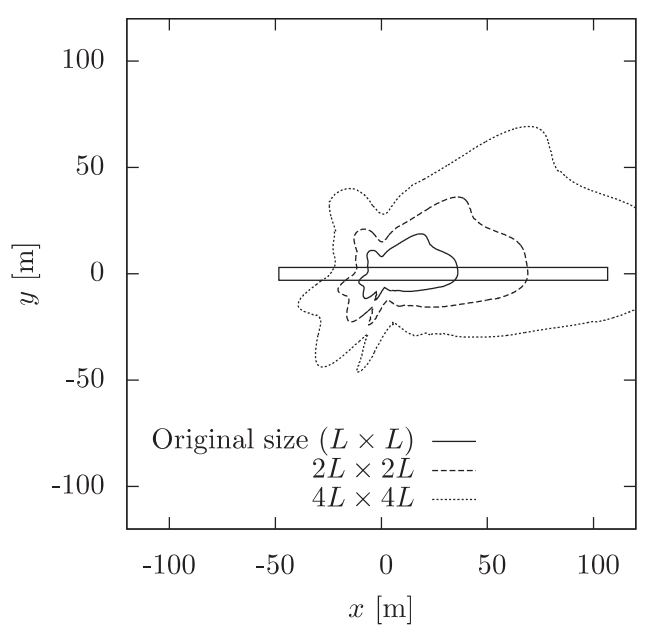

Fig. 7. Disease severity predictions $\left(S_{L}\right)$ using the theoretical model including lateral dispersal (equation 13) in the detailed modeling approach with increasing inoculum source area. The solid, dash, and dot lines represent 0.1 lesions/leaf contours for square inoculum source area with length dimensions of $L, 2 L$, and $4 L$, where $L=1.52 \mathrm{~m}$. Only the center portion $(120 \times 120 \mathrm{~m})$ of the larger numerical grid is displayed. The rectangle depicts the experimental plot (mean dimensions of the two replicate plots). The direction to the right of the source is east and is considered downwind in Sackett and Mundt (2005). fungicide efficacy trials), the refined modeling procedure using local, historical wind data, spore settling velocity, and an estimate of source strength (either information on spore production and canopy escape fraction or measurements of spore deposition) provides a two-dimensional picture of potential risk from interplot contamination. For example, Figure 7 shows 0.1 lesion/leaf contours for three runs with increasing inoculum source area using equation 13 in the refined theoretical modeling procedure and the Hermiston 2002 data set.

While the simple theoretical modeling approach ignoring lateral dispersal retains the nice property of being expressed by an explicit functional relation (which, for example, is useful to estimate the speed of dispersive plant disease waves), the refined procedure incorporating lateral dispersal should be preferred for predicting the spatial pattern of disease spread. The main challenges for the application of the theoretical model to predict spore deposition and disease spread are the determinations of source strength $\left(Q_{T}=F_{T} L_{y}\right)$ and disease resistance $(R(x, y))$. The theory presented here provides a tool to help establish patterns for these two quantities. As an example, experiments such as those presented by Sackett and Mundt (2005) could be carried out simultaneously in two nearby fields with and without irrigation. Results from such a study would help elucidate the role of water availability on the "disease resistance" parameter.

\section{ACKNOWLEDGMENTS}

This research is supported by the National Science Foundation grant AGS1005363. We thank D. E. Aylor, F. J. Ferrandino, J. V. Hayes, C. C. Mundt, E. C. Ogden, G. S. Raynor, and K. E. Sackett for providing the data sets that we used in this study.

\section{LITERATURE CITED}

Aylor, D. E. 1978. Dispersal in time and space: Aerial pathogens. Pages 159-180 in: Plant Disease: An Advanced Treatise. II. J. G. Horsfall and E. B. Cowling, eds. Academic Press, New York.

Aylor, D. E. 1987. Deposition gradients of urediniospores of Puccinia recondite near a source. Phytopathology 77:1442-1448.

Aylor, D. E. 1999. Biophysical scaling and the passive dispersal of fungus spores: Relationship to integrated pest management strategies. Agric. For. Meteorol. 97:275-292.

Aylor, D. E., Fry, W. E., Mayton, H., and Andrade-Piedra, J. L. 2001. Quantifying the rate of release and escape of Phytophthora infestans sporangia from a potato canopy. Phytopathology 91:1189-1196.

Aylor, D. E., and Parlange, J.-Y. 1975. Ventilation required to entrain small particles from leaves. Plant Physiol. 56:97-99.

Aylor, D. E., and Taylor, G. S. 1982. Aerial dispersal and drying of Peronospora tabacina conidia in tobacco shade tents. Proc. Natl. Acad. Sci. USA 79:697-700.

Brutsaert, W. 1982. Evaporation into the Atmosphere: Theory, History, and Applications. Kluwer, Boston.

Chamecki, M. 2012. An analytical solution for dispersion of biological particles emitted from area sources: Inclusion of dispersion in the crosswind direction. Agric. For. Meteorol. 157:30-38.

Chamecki, M., Dufault, N. S., and Isard, S. A. 2012. Atmospheric dispersion of wheat rust spores: A new theoretical framework to interpret field data and estimate downwind dispersion. J. Appl. Meteorol. Climatol. 51:672-685.

Chamecki, M., Gleicher, S., Dufault, N. S., and Isard, S. A. 2011. Diurnal variation in settling velocity of pollen released from maize and consequences for atmospheric dispersion and cross-pollination. Agric. For. Meteorol. 151:1055-1065.

Chamecki, M., and Meneveau, C. 2011. Particle boundary layer above and downstream of an area source: Scaling, simulations, and pollen transport. J. Fluid Mech. 683:1-26.

Cowger, C., Wallace, L. D., and Mundt, C. C. 2005. Velocity of spread of wheat stripe rust epidemics. Phytopathology 95:972-982.

Di-Giovanni, F., Kevan, P. G., and Nasr, M. E. 1995. The variability in settling velocities of some pollen and spores. Grana 34:39-44.

Ferrandino, F. J. 1993. Dispersive epidemic waves: I. Focus expansion within a linear planting. Phytopathology 83:795-802.

Ferrandino, F. J., and Aylor, D. E. 1984. Settling speeds of clusters of spores. Phytopathology 74:969-972. 
Ferrandino, F. J., and Aylor, D. E. 1987. Relative abundance and deposition gradients of clusters of urediniospores of Uromyces phaseoli. Phytopathology 77:107-111.

Frampton, V. L., Linn, M. B., and Hansing, E. E. 1942. The spread of virus diseases of the yellow type under field conditions. Phytopathology 32: 799-808.

Gleicher, S. C., Chamecki, M., Isard, S. A., Pan, Y., and Katul, G. G. 2014. Interpreting three-dimensional spore concentration measurements and escape fraction in a crop canopy using a coupled Eulerian-Lagrangian stochastic model. Agric. For. Meteorol. 194:118-131.

Godson, W. L. 1958. The diffusion of particulate matter from an elevated source. Meteorol. Atmos. Phys. 10:305-327.

Gregory, P. H. 1945. The dispersal of air-borne spores. Trans. Br. Mycol. Soc. 28:26-72.

Gregory, P. H. 1968. Interpreting plant disease dispersal gradients. Annu. Rev. Phytopathol. 6:189-212.

Gregory, P. H. 1973. The Microbiology of the Atmosphere. John Wiley and Sons, New York.

Gregory, P. H., and Read, D. R. 1949. The spatial distribution of insect-borne plant-virus diseases. Ann. Appl. Biol. 36:475-482.

Ingold, C. T. 1967. Liberation mechanisms of fungi. Pages 102-115 in: Airborne Microbes. P. H. Gregory and J. L. Monteith, eds. Cambridge University, London, U.K.

Katul, G. G., Porporato, A., Nathan, R., Siqueira, M., Soons, M. B., Poggi, D., Horn, H. S., and Levin, S. A. 2005. Mechanistic analytical models for longdistance seed dispersal by wind. Am. Nat. 166:368-381.

Legg, B. J., and Powell, F. A. 1979. Spore dispersal in a barley crop: A mathematical model. Agric. Meteorol. 20:47-67.

Loubet, B., Jarosz, N., Saint-Jean, S., and Huber, L. 2007. A method for measuring the settling velocity distribution of large biotic particles. Aerobiologia 23:159-169.

Madden, L. V., Hughes, G., and van den Bosch, F. 2007. The Study of Plant Disease Epidemics. The American Phytopathological Society, St. Paul, MN.

Minogue, K. P. 1989. Diffusion and spatial probability models for disease spread. Pages 127-143 in: Spatial Components of Plant Disease Epidemics. M. J. Jeger and P. Hall, eds. Prentice Hall, Englewood Cliffs, NJ.

Minogue, K. P., and Fry, W. E. 1983a. Models for the spread of disease: Model description. Phytopathology 73:1168-1173.
Minogue, K. P., and Fry, W. E. 1983b. Models for the spread of disease: Some experimental results. Phytopathology 73:1173-1176.

Mundt, C. C. 1989. Use of the modified Gregory model to describe primary disease gradients of wheat leaf rust produced from area sources of inoculum. Phytopathology 79:241-246.

Mundt, C. C., and Leonard, K. J. 1985. A modification of Gregory's model for describing plant disease gradients. Phytopathology 75:930-935.

Mundt, C. C., Sackett, K. E., Wallace, L. D., Cowger, C., and Dudley, J. P. 2009. Long-distance dispersal and accelerating waves of disease: Empirical relationships. Am. Nat. 173:456-466.

Nathan, R., Katul, G. G., Horn, H. S., Thomas, S. M., Oren, R., Avissar, R., Pacala, S. W., and Levin, S. A. 2002. Mechanisms of long-distance dispersal of seeds by wind. Nature 418:409-413.

Pan, Y., Chamecki, M., and Isard, S. A. 2013. Dispersion of heavy particles emitted from area sources in the unstable atmospheric boundary layer. Boundary-Layer Meteorol. 146:235-256.

Pan, Y., Chamecki, M., and Isard, S. A. 2014. Large-eddy simulation of turbulence and particle dispersion inside the canopy roughness sublayer. J. Fluid Mech. 753:499-534.

Raynor, G. S., Hayes, J. V., and Ogden, E. C. 1970. Experimental data on dispersion and deposition of timothy and corn pollen from known sources. BNL Report 50266 (T-595). Brookhaven National Laboratory, Upton, NY.

Reynolds, A. M. 2011. Exponential and power-law contact distributions represent different atmospheric conditions. Phytopathology 101:1465-1470.

Rounds, W. 1955. Solutions of the two-dimensional diffusion equations. Trans. Am. Geophys. Union 36:395-405.

Sackett, K. E., and Mundt, D. D. 2005. Primary disease gradients of wheat stripe rust in large field plots. Phytopathology 95:983-991.

Sutton, O. G. 1932. A theory of eddy diffusion in the atmosphere. Proc. R. Soc. London A 135:143-165.

Van den Bosch, F., Zadoks, J. C., and Metz, J. A. J. 1988. Focus expansion in plant disease. I: The constant rate of focus expansion. Phytopathology 78:54-58.

Van der Plank, J. E. 1967. Spread of plant pathogens in space and time. Pages 227-246 in: Airborne Microbes. P. H. Gregory and J. L. Monteith, eds. Cambridge University, London, U.K.

Van der Plank, J. E. 1975. Principles of Plant Infection. Academic Press, London, U.K. 